\title{
Changes to rehabilitation after total knee replacement
}

Larissa Sattler, Wayne Hing,

Christopher Vertullo

\begin{abstract}
Background
General practitioners play a vital and increasing part in the perioperative care of patients undergoing total knee replacement (TKR). Rising obesity rates, sports-related injuries and an ageing population are likely to result in a sharp increase in TKR procedures within the next decade, combined with higher cost concerns. Rehabilitation practices that show economic efficiency and produce superior patient outcomes are a major focus of current research.
\end{abstract}

\section{Objective}

The aim of this article is to provide an evidence-based summary of current rapid recovery protocols following TKR surgery.

\section{Discussion}

Rapid recovery protocols have been shown to be effective at reducing length of stay, postoperative pain and complications without compromising patient safety. These rapid recovery protocols include same-day mobilisation; blood preservation protocols; selfdirected pedalling-based rehabilitation; and individualised targeted discharge to self-directed, outpatient therapistdirected or inpatient therapist-directed rehabilitation. Low-cost self-directed rehabilitation should be considered usual care, with inpatient rehabilitation reserved for the minority of at-risk patients.
THE BURDEN OF KNEE OSTEOARTHRITIS in Australia is projected to rise in response to an increase in the ageing population, obesity rates and sports-related injuries. ${ }^{1}$ Osteoarthritis now affects one in eleven Australian individuals ${ }^{2}$ and has been reported as the second most common diagnosis made by general practitioners (GPs) consulting with older patients. ${ }^{3,4}$ In accordance with the growth in prevalence of knee osteoarthritis, total knee replacement (TKR) surgery is also forecast to substantially increase in Australia, by up to $276 \%$ by 2030 . $^{1}$

TKR has been proven to be effective for improving a patient's functional status and overall health quality, and it is widely regarded as a highly cost-effective intervention for end-stage knee osteoarthritis. ${ }^{5}$ GP input is vital in the continuum of care for the periods both before and after TKR surgery, particularly for communicating with patients about their expectations for TKR outcomes and rehabilitation. Knee rehabilitation practices have vastly evolved in the past decade, with many being associated with a paradigm shift from a traditional 'sick patient' model of care to a 'well patient' model. ${ }^{6}$ Advances in multimodal pain management, blood management and early mobilisation protocols all contribute to a rapid recovery pathway that has been proven to lower hospital length of stay without adversely affecting postoperative complications or readmissions. ${ }^{7}$ The aim of this narrative review is to provide a summary of the current advancements in rapid recovery following TKR surgery, including early mobilisation, exercise therapy, length of stay and discharge destination.

\section{Perioperative advancements}

Rapid recovery pathways encompass each stage of the patient journey and are primarily aimed towards reducing length of hospital stay while maintaining patient outcomes. ${ }^{8}$ Recent non-surgical advances in the perioperative period have enabled early mobilisation and have had a direct impact on reducing length of stay. Examples of these advancements include blood preservation protocols, multimodal analgesia delivery, avoidance of surgical drains and a decreased use of indwelling urinary catheters (IDCs). Literature suggests that these low-technology, low-cost or no-cost changes in practice account for the greatest share of improvements in patient experience over the past decade. ${ }^{6}$

Minimising perioperative blood loss and reducing postoperative blood transfusion is an effective blood management strategy that is essential to rapid recovery joint arthroplasty and reduces episode-ofcare costs. ${ }^{6}$ Tranexamic acid (TXA) is an antifibrinolytic inhibitor that minimises bleeding by blocking blood clot degradation. ${ }^{9}$ Randomised trials have shown that TXA administered either intravenously or topically results 
in reduced rates of transfusion and thrombotic events in a TKR population, as well as reduced drain output (when used) and increased postoperative haemoglobin..$^{9,10}$ This reduced bleeding has negated the use of surgical drains, with many studies showing no benefit or increased risk with drains. ${ }^{11}$ Moreover, reduced bleeding results in avoidance of blood transfusions, which are costly and increase both infection risk and the length of stay.

In accordance with the decreased need for wound drains is the reduction of IDC use in TKR surgery as a result of low-dose spinal anaesthesia and early mobilisation protocols. Discontinuing routine IDC insertion for TKR is an important component of rapid recovery pathways, which aim to facilitate early mobilisation and achievement of discharge goals. The use of an IDC can potentially increase postoperative urinary tract infections (UTIs), and the duration of its use is the most important risk factor for developing a UTI. ${ }^{12}$ This is highly relevant to patients with TKRs as a UTI has been associated with an increased risk of prosthetic joint infection. ${ }^{13}$ Evidence now suggests that even those patients with TKRs who undergo epidural analgesia show no increased risk of postoperative urological complications without the placement of a preoperative IDC; for those with prostate disorders, the placement of a preoperative IDC increases the risk of postoperative UTI. ${ }^{13}$

Postoperative pain following TKR surgery is feared by a number of patients and, if severe, may inhibit early mobilisation and knee range-of-motion exercises. ${ }^{14}$ Multimodal analgesia is an integral part of a rapid recovery pathway, with regional nerve blocks and peri-articular injections (PAIs) used in favour of epidural anaesthesia or patient-controlled analgesia (PCA) devices. ${ }^{14}$ Low-dose spinal anaesthesia and sedation when combined with a regional nerve block and PAIs is highly effective for promoting rapid recovery. It facilitates early mobilisation without the undesirable side effects of nausea, vomiting and drowsiness often associated with opioid drugs administered by means of intravenous PCA..$^{8,14,15}$
Other expensive high-technological advances such as guided-arm surgery (robotic) and patient-specific instruments, despite heavy promotion and industry claims, have not yet resulted in improved patient outcomes. ${ }^{16}$

\section{Early mobilisation and exercise therapy}

Current definitions of early mobilisation refer to ambulation 4-8 hours following TKR surgery. ${ }^{17,18}$ 'Day of surgery' mobilisation is becoming a more common practice in Australia because of the wide range of health benefits it provides to patients, ${ }^{19}$ with the realisation that it is extremely advantageous for patients to gain consciousness post-surgery with no pain and the ability to mobilise within a few hours of their surgery. A literature review of early mobilisation protocols in a post-surgical population revealed reduced rates of postoperative complications such as deep vein thrombosis, pneumonia, atelectasis, UTIs, sepsis, myocardial infarction and stroke. ${ }^{20}$ Early mobilisation has shown a reduction in hospital length of stay, ${ }^{21,22}$ and there is evidence to suggest it can provide improved patient outcomes without an increased risk of complications. ${ }^{23,24}$ Patients have also reported an overall increase in satisfaction with the introduction of early mobilisation and less pain than patients receiving standard care. ${ }^{25}$ Previous attitudes about hospitalisation have reinforced passive coping strategies and inactivity in patients; however, early mobilisation assists to counteract this and improve health-related quality of life..$^{23,26}$

Despite patients with TKRs receiving an in-hospital physiotherapy program of some description, the optimum type of TKR exercise intervention in the early postoperative phase remains unknown. ${ }^{27-29}$ A recent systematic literature review and meta-analysis exposed an urgent need for further high-quality studies into supervised exercise therapy programs that aim to provide greater functional outcomes and patient-reported satisfaction following TKR surgery, particularly in the early postoperative period. ${ }^{27}$ Differing protocols provide some instruction for exercises after TKR to be used in combination with healthcare professionals' clinical judgment to make adjustments or progressions. ${ }^{29}$ Unfortunately, this paucity of evidence hinders the creation of best-practice guidelines for specific exercises, including their duration or frequency following TKR surgery; currently, vast variations in care exist. ${ }^{28}$ The goals of physiotherapy have changed in line with rapid recovery pathways that have been shown to rapidly decrease length of stay after TKR surgery. The most effective physiotherapy exercise protocol during the acute hospital stay is based on restoring safe functional mobility and performing exercises with a focus on increasing knee range-of-motion such as bike pedalling (Figures 1-3). ${ }^{30}$ In 2019, a randomised controlled trial investigated early exercise therapy protocols after TKR surgery and was published in the Journal of Bone and Joint Surgery. It showed that a simple self-directed, low-cost, three-exercise bike pedalling-based protocol was superior to a therapist-led standard multi-exercise regimen, for both functional and patientreported outcomes. ${ }^{30}$ Based on the results of this trial, patients who have had a TKR can complete a few simple home-based exercises - focusing on bike pedalling, knee extension and heel-toe walking practice - for the first two weeks following their knee surgery and expect optimal outcomes. Following this early recovery

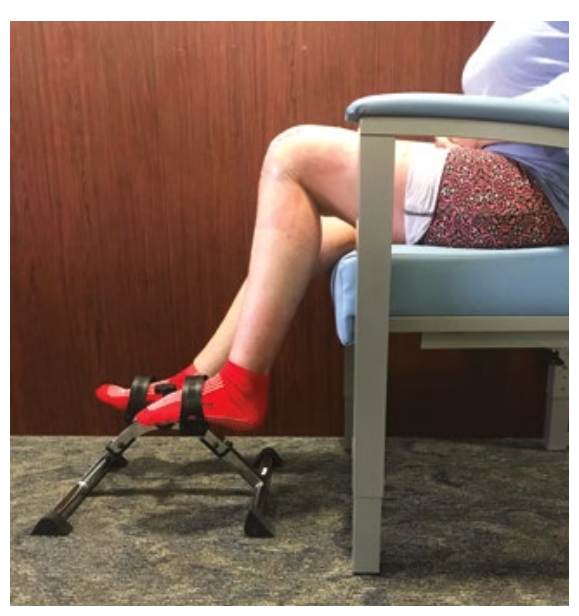

Figure 1. Stationary floor pedal exerciser 
period, patients yet to gain 90-degree knee flexion or those who are having difficulty progressing with their mobility may benefit from some supervised physiotherapy treatment, with no significant difference shown between patients supervised in the home or outpatient setting. ${ }^{31}$

\section{Length of stay, outpatient arthroplasty and discharge destination}

Reducing length of stay has been shown to improve functional recovery and enable a more rapid return to independent living; however, early concerns have been raised that decreasing the duration of hospital stay may result in increased hospital readmissions. ${ }^{32}$ Numerous studies have now shown that rapid recovery programs can shorten length of stay without increasing complications or readmissions. ${ }^{7,33-35}$ Length of stay duration after TKR surgery is decreasing; in Australia, the average length of stay after TKR surgery declined from 7.1 days in 2009 to 5.4 days in $2016 .{ }^{36}$ This decrease in length of stay aligns with trends in the USA where, from 2002 to 2013, the mean inpatient period postTKR surgery decreased from 4.06 days to 2.97 days, and the percentage of hospital inpatient periods of $\geq 5$ days decreased from $24.7 \%$ to $6.1 \% .{ }^{37}$ With concerns regarding healthcare expenditure remaining at the national forefront, a new model of care is arising: outpatient or day procedure arthroplasty. Outpatient arthroplasty is an appealing option for carefully selected patient populations, and it relies on structured rapid recovery pathways, including preoperative patient education, regional anaesthesia and early mobilisation. ${ }^{37}$ Although the uptake of outpatient arthroplasty in Australia has been slower than in the USA, ${ }^{38}$ discussions about healthcare reform and a growing body of evidence showing its safety in certain patient populations mean that it may become a viable way to reduce the growing financial burden for the predicted increase in TKR surgery over the next decade. ${ }^{39}$

Referral rates to extended inpatient rehabilitation in Australia are high. It is estimated that approximately $40 \%$ of patients who are privately insured and $20 \%$ of patients in the public sector were referred to inpatient rehabilitation following TKR surgery in $2014 .{ }^{40}$ This high referral pattern contrasts with evidence to suggest that functional outcomes for those attending inpatient rehabilitation are not superior in randomised controlled trials to those discharged to home or community-based rehabilitation. ${ }^{40,41}$ A recent landmark Australian study, the HIHO randomised clinical trial, compared outcomes for patients with TKRs discharged to inpatient rehabilitation with those who completed a supervised home-based program, finding no differences between groups for mobility, reported pain and function, or quality of life. ${ }^{40}$ Although a formal cost analysis was not performed in this trial, the results suggest that healthcare system costs can be reduced without harming patient outcomes. ${ }^{36}$ Literature states that a home discharge can be $\$ 16,000$ less expensive than discharge to an acute inpatient rehabilitation facility; given this potential

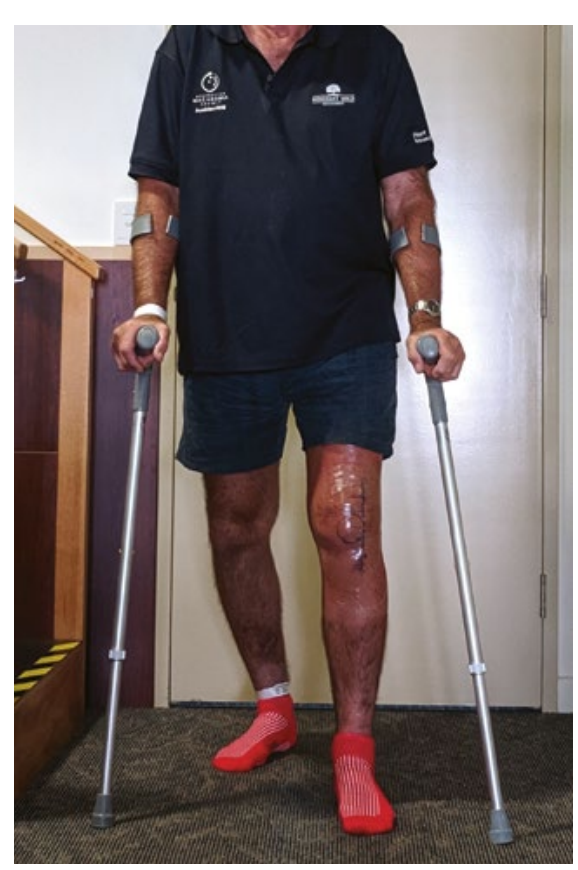

Figure 2. Gait retraining on the first postoperative day following total knee replacement surgery cost saving, there needs to be urgent review of the reasons behind the large number of rehabilitation referrals..$^{41,42}$ The seemingly indiscriminate use of inpatient rehabilitation following uncomplicated primary TKR surgery is now being considered as 'low-value care' in Australia. However, system-level changes to guidelines and incentives for hospitals as well as development of more accurate patient screening tools will be needed if a meaningful transformation to current practice is to be made. ${ }^{36}$

\section{Conclusion}

The use of rapid recovery pathways after TKR surgery is increasing. They have been shown to be effective at reducing length of stay without compromising patient safety. Examples of rapid recovery protocols include perioperative advancements, early mobilisation and active exercise therapy such as bike pedalling and regaining functional mobility, which in turn has also contributed to a further reduction in length of stay. Financial considerations

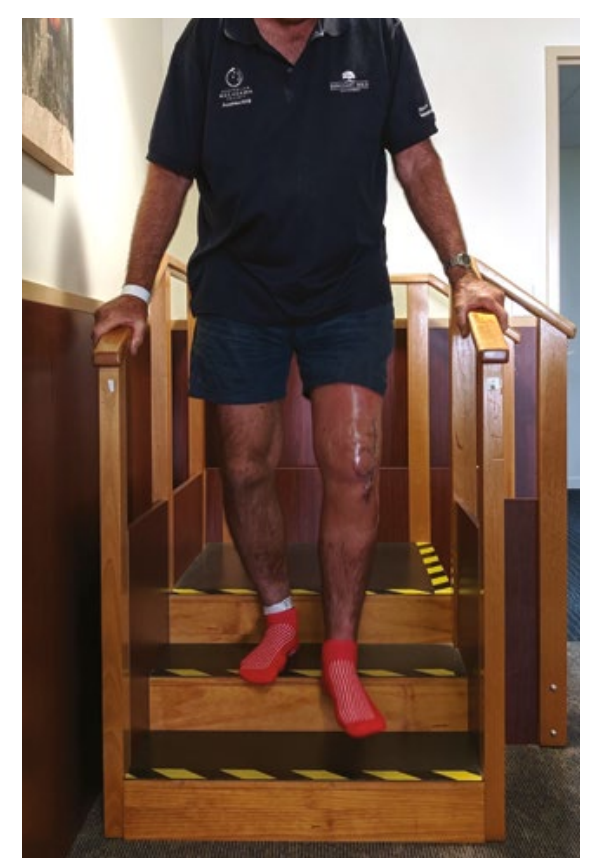

Figure 3. Stair climb practice on the first postoperative day following total knee replacement surgery 
need to be of urgent priority, as the economic burden of TKR will continue to strain both the private and public sectors as rates of TKR surgery are forecast to increase. Recently, it has been shown that inpatient rehabilitation after routine TKR surgery offers no greater functional or quality of life benefits than discharge to home. Healthcare reform and hospital policy review is needed to better facilitate home-based care. Additionally, outpatient arthroplasty - which would currently be considered novel in Australia - is rapidly growing overseas and may provide a significant cost-saving option for eligible patients.

\section{Key points}

- Rehabilitation after TKR surgery is moving to an individualised stratified model, with low-cost self-directed home-based rehabilitation as the standard and adjuvant therapist-directed inpatient or outpatient rehabilitation as needed.

- Low-cost perioperative interventions such as blood preservation with TXA and early mobilisation analgesia have enabled rapid recovery pathways, while more expensive technology driven interventions have not.

- With the introduction of same-day joint surgery in Australia for low-risk individuals, length of stay after TKR surgery will continue to reduce.

- Perioperative advancements in TKR surgery and rehabilitation have resulted in shorter hospital stays and faster recovery with less pain, which is relevant to discussions between GPs and their patients with TKRs.

\footnotetext{
Authors

Larissa Sattler MPhtySt, Assistant Professor, Physiotherapy, Faculty of Health Sciences \& Medicine, Bond University, Qld. Isattler@bond.edu.au Wayne Hing PhD, Head of Physiotherapy, Faculty of Health Sciences and Medicine, Bond University, Qld Christopher Vertullo MBBS, PhD, FRACS (Orth), FAOrthA, Director, Knee Research Australia, Qld Competing interests: None.

Funding: CV reports grants from Ramsay Health and Allocuro, and personal fees from Arthrex, outside the submitted work.

Provenance and peer review: Commissioned, externally peer reviewed.
}

\section{References}

1. Ackerman IN, Bohensky MA, Zomer E, et al. The projected burden of primary total knee and hip replacement for osteoarthritis in Australia to the year 2030. BMC Musculoskelet Disord 2019;20(1):90. doi: 10.1186/s12891-019-2411-9.

2. Arthritis Australia Models of Care Steering Group. Time to move: Osteoarthritis A national strategy to reduce a costly burden. Glebe, NSW: Arthritis Australia, 2014

3. Peat G, McCarney R, Croft P. Knee pain and osteoarthritis in older adults: A review of community burden and current use of primary health care. Ann Rheum Dis 2001;60(2):91-97. doi: 10.1136/ard.60.2.91.

4. Arthritis Research UK National Primary Care Centre. Musculoskeletal matters bulletin 1: What do general practitioners see? Staffordshire, UK: Keele University, 2009.

5. Ho DM, Huo MH. Are critical pathways and implant standardization programs effective in reducing costs in total knee replacement operations? J Am Coll Surg 2007;205(1):97-100. doi: 10.1016/j.jamcollsurg.2007.03.009.

6. Sculco PK, Pagnano MW. Perioperative solutions for rapid recovery joint arthroplasty: Get ahead and stay ahead. J Arthroplasty 2015;30(4):518-20. doi: 10.1016/j.arth.2015.01.036.

7. Sibia US, Waite KA, Callanan MA, Park AE, King PJ, MacDonald JH. Do shorter lengths of stay increase readmissions after total joint replacements? Arthroplast Today 2017;3(1):51-55. doi: 10.1016/j.artd.2016.05.001.

8. Galbraith AS, McGloughlin E, Cashman J. Enhanced recovery protocols in total joint arthroplasty: A review of the literature and their implementation. Ir J Med Sci 2018;187(1):97-109. doi: 10.1007/s11845-017-1641-9.

9. Kelley TC, Tucker KK, Adams MJ, Dalury DF. Use of tranexamic acid results in decreased blood loss and decreased transfusions in patients undergoing staged bilateral total knee arthroplasty. Transfusion 2014;54(1):26-30. doi: 10.1111/trf.12167.

10. Abdel MP, Chalmers BP, Taunton MJ, et al. Intravenous versus topical tranexamic acid in total knee arthroplasty: Both effective in a randomized clinical trial of 640 patients. J Bone Joint Surg Am 2018;100(12):1023-29. doi: 10.2106/JBJS.17.00908.

11. Kim TK, Chang CB, Koh IJ. Practical issues for the use of tranexamic acid in total knee arthroplasty: A systematic review. Knee Surg Sports Traumatol Arthrosc 2014;22(8):1849-58.

12. Ma Y, Lu X. Indwelling catheter can increase postoperative urinary tract infection and may not be required in total joint arthroplasty: A metaanalysis of randomized controlled trial. BMC Musculoskelet Disord 2019;20(1):11. doi: 10.1186/ s12891-018-2395-x.

13. Scotting OJ, North WT, Chen C, Charters MA Indwelling urinary catheter for total joint arthroplasty using epidural anesthesia. J Arthroplasty 2019;34(10):2324-28. doi: 10.1016/j.arth.2019.05.047.

14. Song $\mathrm{MH}, \mathrm{Kim} \mathrm{BH}$, Ahn SJ, et al. Peri-articular injections of local anaesthesia can replace patientcontrolled analgesia after total knee arthroplasty: A randomised controlled study. Int Orthop 2016;40(2):295-99. doi: 10.1007/s00264-0152940-2.

15. Buckenmaier CC, 3rd. Anaesthesia for outpatient knee surgery. Best Pract Res Clin Anaesthesiol 2002;16(2):255-70. doi: 10.1053/bean.2002.0237.

16. Dorr LD. CORR Insights ${ }^{\oplus:}$ : Does robotic-assisted TKA result in better outcome scores or long- term survivorship than conventional TKA? A randomized, controlled trial. Clin Orthop Relat Res 2020;478(2):276-78. doi: 10.1097/ CORR.0000000000000969.

17. Castorina S, Guglielmino C, Castrogiovanni P, et al. Clinical evidence of traditional vs fast track recovery methodologies after total arthroplasty for osteoarthritic knee treatment. A retrospective observational study. Muscles Ligaments Tendons J 2018;7(3):504-13. doi: 10.11138/mltj/2017.7.3.504.

18. Warwick H, George A, Howell C, Green C, Seyler TM, Jiranek WA. Immediate physical therapy following total joint arthroplasty: Barriers and impact on short-term outcomes. Adv Orthop 2019;6051476. doi: 10.1155/2019/6051476.

19. Chua MJ, Hart AJ, Mittal R, Harris IA, Xuan W, Naylor JM. Early mobilisation after total hip or knee arthroplasty: A multicentre prospective observational study. PLoS One 2017;12(6):e0179820. doi: 10.1371/journal. pone.0179820.

20. Epstein NE. A review article on the benefits of early mobilization following spinal surgery and other medical/surgical procedures. Surg Neurol Int 2014;5(Suppl 3):S66-S73. doi: 10.4103/21527806.130674.

21. Renkawitz T, Rieder T, Handel M, et al. Comparison of two accelerated clinical pathways After total knee replacement how fast can we really go? Clin Rehabil 2010;24(3):230-39. doi: 10.1177/0269215509353267.

22. Husted $\mathrm{H}$, Hansen $\mathrm{HC}$, Holm G, et al. What determines length of stay after total hip and knee arthroplasty? A nationwide study in Denmark. Arch Orthop Trauma Surg 2010;130(2):263-68. doi: 10.1007/s00402-009-0940-7.

23. Winther SB, Foss OA, Wik TS, et al. 1-year follow-up of 920 hip and knee arthroplasty patients after implementing fast-track. Acta Orthop 2015;86(1):78-85. doi: 10.3109/17453674.2014.957089.

24. Schneider M, Kawahara I, Ballantyne G, et al. Predictive factors influencing fast track rehabilitation following primary total hip and knee arthroplasty. Arch Orthop Trauma Surg 2009;129(12):1585-91. doi: 10.1007/s00402-0090825-9.

25. Cox J, Cormack C, Prendergast M, Celestino H, Willis S, Witteveen M. Patient and provider experience with a new model of care for primary hip and knee arthroplasties. Int J Orthop Trauma Nurs 2016;20:13-27. doi: 10.1016/j. ijotn.2015.05.003.

26. van der Sluis G, Goldbohm RA, Bimmel R, Galindo Garre F, Elings J, Hoogeboom TJ, van Meeteren NLU. What augmented physical activity and empowerment can bring to patients receiving total knee replacement: Content, implementation, and comparative effectiveness of a new function-tailored care pathway in a routine care setting. Biomed Res Int 2015;745864. doi: 10.1155/2015/745864.

27. Sattler LN, Hing WA, Vertullo CJ. What is the evidence to support early supervised exercise therapy after primary total knee replacement? A systematic review and meta-analysis. BMC Musculoskelet Disord 2019;20(1):42. doi: 10.1186/ s12891-019-2415-5.

28. Naylor J, Harmer A, Fransen M, Crosbie J, Innes L. Status of physiotherapy rehabilitation after total knee replacement in Australia. Physiother Res Int 2006;11(1):35-47. doi: 10.1002/pri.40.

29. Mistry JB, Elmallah RD, Bhave A, et al. Rehabilitative guidelines after total knee arthroplasty: A review. J Knee Surg 2016;29(3):201-17. doi: 10.1055/s-0036-1579670. 
30. Sattler LN, Hing WA, Vertullo CJ. Pedaling-based protocol superior to a 10-exercise, non-pedaling protocol for postoperative rehabilitation after total knee replacement: A randomized controlled trial. J Bone Joint Surg Am 2019;101(8):688-95. doi: 10.2106/JBJS.18.00898.

31. Artz N, Elvers KT, Lowe CM, Sackley C, Jepson P, Beswick AD. Effectiveness of physiotherapy exercise following total knee replacement: Systematic review and meta-analysis. BMC Musculoskelet Disord 2015;16:15. doi: 10.1186/ s12891-015-0469-6.

32. Yakkanti RR, Miller AJ, Smith LS, Feher AW, Mont MA, Malkani AL. Impact of early mobilization on length of stay after primary total knee arthroplasty. Ann Transl Med 2019;7(4):69.

33. Berg U, BüLow E, Sundberg M, Rolfson O. No increase in readmissions or adverse events after implementation of fast-track program in total hip and knee replacement at 8 Swedish hospitals: An observational before-andafter study of 14,148 total joint replacements 2011-2015. Acta Orthop 2018;89(5):522-27. doi: 10.1080/17453674.2018.1492507.

34. Barad SJ, Howell SM, Tom J. Is a shortened length of stay and increased rate of discharge to home associated with a low readmission rate and cost-effectiveness after primary total knee arthroplasty? Arthroplast Today 2018;4(1):107-12. doi: 10.1016/j.artd.2015.08.003.
35. Sarpong NO, Boddapati V, Herndon CL, Shah RP, Cooper HJ, Geller JA. Trends in length of stay and 30-day complications after total knee arthroplasty: An analysis from 2006 to 2016. J Arthroplasty 2019;34(8):1575-80. doi: 10.1016/j. arth.2019.04.027.

36. Schilling C, Keating C, Barker A, Wilson SF Petrie D. Predictors of inpatient rehabilitation after total knee replacement: An analysis of private hospital claims data. Med J Aust 2018;209(5):222-27. doi: 10.5694/mja17.01231.

37. Molloy IB, Martin BI, Moschetti WE, Jevsevar DS. Effects of the length of stay on the cost of total knee and total hip arthroplasty from 2002 to 2013. J Bone Joint Surg Am 2017;99(5):402-07. doi: 10.2106/JBJS.16.00019.

38. Klingenstein GG, Schoifet SD, Jain RK, Reid JJ, Porat MD, Otegbeye MK. Rapid discharge to home after total knee arthroplasty is safe in eligible Medicare patients. J Arthroplasty 2017;32(11):3308-13. doi: 10.1016/j. arth.2017.06.034

39. Jaibaji M, Volpin A, Haddad FS, Konan S. Is outpatient arthroplasty safe? A systematic review. J Arthroplasty 2020;35(7):1941-49. doi: 10.1016/j. arth.2020.02.022

40. Buhagiar MA, Naylor JM, Harris IA, et al. Effect of inpatient rehabilitation vs a monitored home- based program on mobility in patients with total knee arthroplasty: The HIHO randomized clinical trial. JAMA 2017;317(10):1037-46. doi: 10.1001/ jama.2017.1224.

41. Mayer MA, Pirruccio K, Sloan M, Sheth NP. Discharge home is associated with decreased early complications following primary total joint arthroplasty. J Arthroplasty 2019;34(11):2586-93. doi: 10.1016/j.arth.2019.06.049.

42. Mahomed NN, Davis AM, Hawker G, et al. Inpatient compared with home-based rehabilitation following primary unilateral total hip or knee replacement: A randomized controlled trial. J Bone Joint Surg Am 2008;90(8):1673-80. doi: 10.2106/JBJS.G.01108.

correspondence ajgp@racgp.org.au 